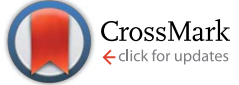

Cite this: RSC Adv., 2016, 6, 41991

\title{
Modulation of singlet oxygen generation in halogenated BODIPY dyes by substitution at their meso position: towards a solvent-independent standard in the vis region $\uparrow$
}

\author{
Nerea Epelde-Elezcano, ${ }^{2}$ Virginia Martínez-Martínez, ${ }^{* a}$ Eduardo Peña-Cabrera, ${ }^{b}$ \\ César F. A. Gómez-Durán, ${ }^{b}$ Iñigo López Arbeloa ${ }^{a}$ and Sylvie Lacombe ${ }^{c}$
}

\begin{abstract}
A series of halogenated BODIPY with different substitutions patterns at meso position such as aryl groups and heteroatoms (methylthio (MeS) and amino $\left(\mathrm{NR}_{2}\right)$ groups) with various electron-donor and electronwithdrawing properties are studied. A detailed analysis of their photophysical properties as well as a thorough determination of their singlet oxygen quantum yields enlightens the role of the substituents at the meso position. Importantly, one of the new halogenated BODIPY with a methylthio group at the meso position, with intense absorption band in the visible region is proposed as a suitable reference with high singlet oxygen quantum yield, practically constant in different solvents of a wide polarity range.
\end{abstract}

Received 4th March 2016 Accepted 20th April 2016

DOI: $10.1039 / \mathrm{c} 6 \mathrm{ra05820e}$

www.rsc.org/advances

complexity, low molar extinction coefficients in the red-part of the visible spectra, poor or low specific accumulation in tumour tissue. Therefore high doses and long time between administration of drug and irradiation are needed. Moreover, their extremely long clearance from the body may produce high cutaneous photosensitivity post-treatment and other side effects. ${ }^{2,5-7}$

Recently, a new type of chromophores, 4,4-difluoro-4-bora$3 a, 4 a$-diaza-s-indacene, also known as BODIPY (used in the following) have attracted considerable attention because of their very favorable chemico-physical features, including high extinction coefficients in the visible region and resistance to photobleaching. ${ }^{8}$ Besides, their properties are practically unaffected by environmental conditions (solvent polarity or $\mathrm{pH}$ ) and by the high lipophilicity of the medium. ${ }^{9}$ Moreover their synthesis versatility allows an exhaustive and selective structural modification/functionalization around the core structure of the chromophore, in order to modulate their properties and to enhance their therapeutic efficiency. Although BODIPYs usually exhibit negligible efficiency of triplet $\mathrm{T}$ formation due to their high fluorescence quantum yields, there are different tactics to enhance the intersystem crossing to the triplet state such as the incorporation of heavy atoms (i.e. Br, I) directly to the BODIPY core, thereby favoring spin-orbit coupling., ${ }^{\mathbf{6} 10-13}$ Indeed, the in vitro or in vivo tests have already showed the efficacy of halogenated BODIPYs to kill tumor cells. ${ }^{\mathbf{1 4}}$

In this work new halogenated-BPD with different substitution patterns at their meso position are studied (Scheme 1). Particularly, several halogenated BODIPY with different halide atoms $(\mathrm{Br}$ or I) at 2,6 positions and different substitutions patterns at meso (or atom number 8) position such as $p$-substituted phenyl groups and heteroatoms such as methylthio (MeS) and amino groups

\footnotetext{
${ }^{a}$ Molecular Spectroscopy Laboratory, University of the Basque Country UPV/EHU, Apartado 644, 48080 Bilbao, Spain.E-mail: virginia.martinez@ehu.eus

${ }^{b}$ Chemistry Department, University of Guanajuato, Noria Alta S/N 36050, Mexico 'IPREM UMR CNRS 5254, Université de Pau et des Pays de l'Adour, Hélioparc, 2 av. Président Angot, 64053 Pau Cedex 09, France

$\dagger$ Electronic supplementary information (ESI) available: The rotamers and the resonance structures of the different BODIPYs, the description of the singlet oxygen calculation by direct and indirect method, an illustrative scheme of the processes involved after the excitation and the variation of ${ }^{1} \mathrm{O}_{2}$ emission signal with the concentration of 7 and 8 compounds. See DOI: 10.1039/c6ra05820e
} 


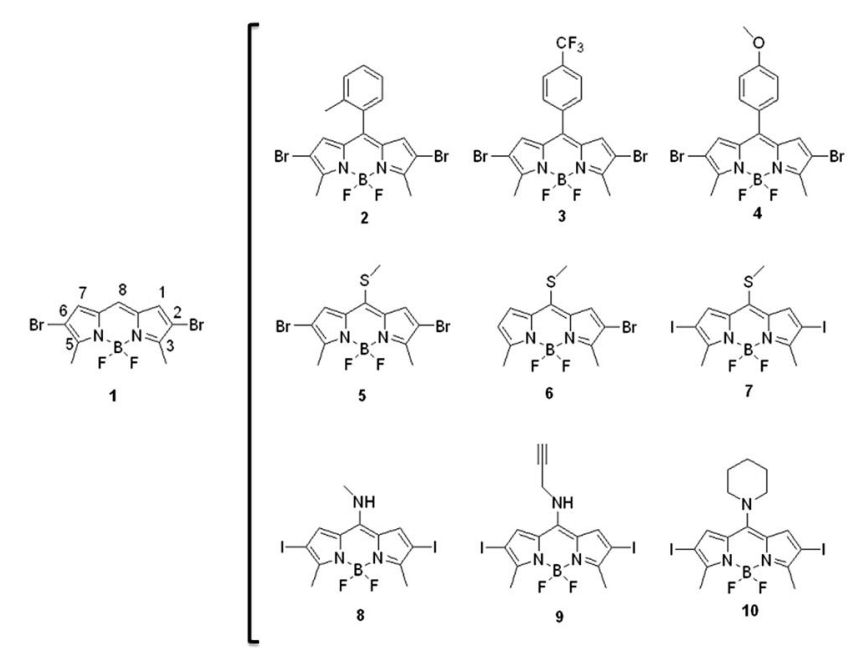

Scheme 1 The molecular structure of halogenated BODIPY derivatives.

$\left(\mathrm{NR}_{2}\right)$ are considered. As previously described for nonhalogenated BODIPY, ${ }^{15-17}$ the absorption and emission bands are expected to shift hypsochromically or bathochromically depending on the substituted heteroatom at the 8-position. This work is thus mainly focused on the influence of different substitution patterns of several halogenated BODIPY on the singlet oxygen production, as well as their photophysical properties.

In order to determine the absolute singlet oxygen quantum yields $\left(\Phi_{\Delta}\right)$ of novel sensitizers different approaches may be used: (a) an indirect method based on reactive and specific chemical probes for singlet oxygen detection or (b) the direct detection of the weak singlet oxygen luminescence in the nearIR region $(1276 \mathrm{~nm})$. The direct method should provide a more straightforward and accurate $\Phi_{\Delta}$ value with respect to the indirect method as long as suitable reference sensitizers are used. Actually, a good reference sensitizer should be thermally and photochemically stable. Its singlet oxygen quantum yield should be precisely known (\% relative error $\leq 10 \%$ ), solventindependent and relatively high $\left(\Phi_{\Delta} \geq 0.40\right)$. Moreover, it should have a low rate constant of singlet oxygen quenching by the sensitizer itself $\left(k_{\mathrm{t}}^{\mathrm{PS}}\right)$, benefiting from the absence of selfsensitization of its own photo-oxidation and enabling the use of rather high sensitizer concentrations. ${ }^{4}$ Ideally, the same excitation wavelength and mainly the same solvent should be used for the reference and for the PS under study. ${ }^{18}$ Among standard photosensitizers for singlet oxygen production, $1 \mathrm{H}$ phenalen-1-one (phenalenone) is considered as the universal reference with a singlet oxygen quantum yield close to 1 , varying only in a narrow range $\left(0.94<\Phi_{\Delta}<1.00\right)$ in numerous polar and non-polar solvents. ${ }^{19}$ However, phenalenone can only be used under UV-excitation light since its absorption bands are in the 200-400 $\mathrm{nm}$ range with molar absorption coefficients around $\varepsilon \sim 10000 \mathrm{M}^{-1} \mathrm{~cm}^{-1}$. On the other hand, photosensitizers from the xanthene (such as Rose Bengal) or phenothiazine (such as methylene blue) family show strong absorption bands in the visible range $\left(\varepsilon>50000 \mathrm{M}^{-1} \mathrm{~cm}^{-1}\right)$ and are commonly used as standards for the singlet oxygen production in the vis region. However, they are only suitable in polar solvents such as in ACN, EtOH or $\mathrm{MeOH}$, with sometimes very scattered published singlet oxygen quantum yields (i.e. for $\mathrm{RB}$ in $\left.\mathrm{ACN}, \Phi_{\Delta}=0.5-0.8\right) .{ }^{3,18}$ Moreover, these reference PSs undergo prompt photo-bleaching, have a high tendency to aggregate in polar solvents and are insoluble in apolar or chlorinated solvents. Therefore, a suitable reference, soluble in both apolar and polar solvents, with high $\Phi_{\Delta}$ and good photostability would be desired for the vis region. In the following, one of the new halogenated BODIPY studied in this work could fulfil all those requirements.

\section{Experimental}

\section{Synthetic procedures and characterization}

Rose Bengal and phenalenone (purity 95\% and 97\%, respectively) were purchased from Sigma-Aldrich and used as received. The synthesis of the new halogenated BODIPYs has been recently detailed in ref. 16 .

Their photophysical properties were measured in diluted solutions (approx. $5 \times 10^{-6} \mathrm{M}$ ). The UV/vis absorption spectra were recorded on a Varian dual beam spectrometer (CARY 4E) in transmittance mode. Emission spectra were recorded on a spectrofluorimeter Edinburgh Instruments (FLSP920 model) with a xenon flash lamp $450 \mathrm{~W}$ as the excitation source. The fluorescence spectra were corrected from the wavelength dependence of the detector sensibility. Fluorescence quantum yield $(\phi)$ was obtained using several references such as the commercial BODIPY 597, $605\left(\phi_{\text {flu }}^{\mathrm{r}}=0.43\right.$ and 0.66 in ethanol, respectively) and cumarine $152\left(\phi_{\text {flu }}^{\mathrm{r}}=0.18\right.$ in ethanol).

The production of singlet oxygen was determined by indirect method, based on the chemical reaction of 9,10-dimethylanthracene (DMA) as a chemical probe (see ESI $\dagger$ for details) ${ }^{20}$ and by direct measurement of the luminescence at $1276 \mathrm{~nm}$ of the singlet oxygen with a NIR detector (InGaAs detector, Hamamatsu G8605-23) integrated in the spectrofluorimeter (Edinburgh Instruments, model FL920). See ESI $\uparrow$ for details on the calculations. In the latter case, the singlet oxygen signal was recorded in cells of $1 \mathrm{~cm}$ in front configuration (front face), $40^{\circ}$ and $50^{\circ}$ to the excitation and emission beams, respectively and leaned $30^{\circ}$ to the plane formed by the direction of incidence. The signal was filtered by a low cut-off filter at $850 \mathrm{~nm}$. The singlet oxygen signal was measured in the BODIPY concentration range $10^{-4}$ to $10^{-5} \mathrm{M}$ with at least five different concentrations. Note here that there was not any indication of BODIPY aggregation since the absorption spectra of the different BODIPYs did not show any modifications as the concentration increased (up to $10^{-4} \mathrm{M}$ ). The singlet oxygen quantum yield $\left(\Phi_{\Delta}\right)$ was calculated using the following equation (subscript $\mathrm{R}$ refers to the reference and PS to the photosensitizer under study):

$$
\phi_{\Delta}=\phi_{\Delta}(R)\left(\frac{\alpha_{\mathrm{R}}}{\alpha_{\mathrm{Ps}}}\right)\left(\frac{\mathrm{S}_{\mathrm{e}}^{\mathrm{PS}}}{\mathrm{S}_{\mathrm{e}}^{\mathrm{R}}}\right)
$$

where $\Phi_{\Delta}(R)$ is the quantum yield of singlet oxygen production of the reference. We used Rose Bengal $\left(\Phi_{\Delta}=0.53\right.$ in acetonitrile, 
measured in our laboratory with respect to phenalenone in acetonitrile $\left.\left(\Phi_{\Delta}=1\right)^{19}\right)$. The factor $\alpha=1-10^{- \text {Abs }}$ corrects the different amount of photons absorbed by the samples, Se is the singlet oxygen signal of the samples. Although, it is highly recommended to carry out the reference and PS quantum yield determination in the same solvent, it is sometimes necessary to use different solvents. In that case, a correction has to be introduced, taking into account $\tau_{\mathrm{P}}^{\Delta}$, lifetime of singlet oxygen phosphorescence and the refractive index of the respective solvents (see ESI, eqn SI $07 \& 08 \dagger$ ). ${ }^{15,21}$ Note here that, in eqn (1) the rate constant of the total quenching of singlet oxygen by the photosensitizer itself $\left(k_{\mathrm{t}}^{\mathrm{PS}}\right)$ is assumed to be negligible relative to the rate constant of quenching of singlet oxygen by the solvent $\left(k_{\mathrm{d}}\right)$ (see ESI, eqn 05 and $06 \dagger$ ).

The lifetime of singlet oxygen in different solvents was measured by the time resolved decay of the phosphorescence signal at $1276 \mathrm{~nm}$ in the same spectrofluorometer (Edinburgh Instruments, model FL920) using a pulsed flash lamp 60 microseconds $\mathrm{W}(\mu \mathrm{F} 920)$ as excitation and recording 5000 counts in the peak emission channel. The singlet oxygen lifetimes $(\tau)$ were obtained from the slope by means of an iterative method by the FAST software (Fluorescent Analysis Software Technology). The goodness of the exponential fit was controlled by statistical parameters (chi-square, Durbin-Watson and the analysis of the residuals).

The setup and method used for the indirect determination of singlet oxygen quantum yield using 9,10-dimethylanthracene (DMA, Sigma Aldrich) as a singlet oxygen probe are described in ESI (eqn SI 08 and SI $11 \dagger$ ). Briefly, the solution of BODIPY 7 in ACN solvent $\left(3 \times 10^{-5} \mathrm{M}\right)$ containing DMA $\left(6 \times 10^{-5} \mathrm{M}\right)$ was excited at the absorption maxima $\left(\lambda_{\text {abs }}=533 \mathrm{~nm}\right)$ using a $200 \mathrm{~W}$ $\mathrm{Xe}-\mathrm{Hg}$ light source equipped with a monochromator.

\section{Results and discussion}

\section{8-Aryl-BODIPYs}

First, the photophysical properties and singlet oxygen production of several BODIPY with halogenated atoms at 2,6 position and different aryl groups at meso (or 8) position were studied in ACN solvent. The results are compared with dye 1 (2,6-diBromoBODIPY) without any substitution at its meso position, considered as the reference compound (Table 1 and Fig. 1).

The photophysical behaviour and more particularly the quantum yields of singlet oxygen production of the reference compound, 1 and of its homologous $\mathbf{2}$ with an ortho-methyl-phenyl group substituted at the meso position are very similar (Table 1).

It has been previously demonstrated that non-halogenated 8aryl-BODIPYs usually present a very low fluorescence quantum yield, attributed to the deactivation by internal conversion due to the free rotational motion of the pendant 8-phenyl group that can also distort the indacene planarity. ${ }^{22-26}$ However the internal conversion is drastically reduced by the substitution in ortho position of the phenyl group (here by a methyl group) because it hampers the free rotation due to sterical hindrance: the aryl group is blocked practically in perpendicular disposition relative to the BODIPY core (Fig. S1†). That means that the substituted aryl group in $\mathbf{2}$ does not take part in the electron
Table 1 Photophysical parameters and singlet oxygen quantum yields of the halogenated-BODIPY derivatives in ACN; $\lambda_{\text {abs }}$ (absorption maxima), $\varepsilon_{\max }$ (molar absorption coefficient), $\lambda_{\text {flu }}$ (fluorescence maxima), $\phi_{\text {flu }}$ (fluorescence quantum yield), $\Phi_{\Delta}$ (singlet oxygen quantum yield), $k_{t}^{\text {PS }}$ (rate constant of singlet oxygen total quenching by the PS)

\begin{tabular}{lllllll}
\hline & $\begin{array}{l}\lambda_{\mathrm{abs}} \\
{[\mathrm{nm}]}\end{array}$ & $\begin{array}{l}\varepsilon_{\text {max }} \\
{\left[10^{4} \mathrm{M} \mathrm{cm}^{-1}\right]}\end{array}$ & $\begin{array}{l}\lambda_{\text {flu }} \\
{[\mathrm{nm}]}\end{array}$ & $\phi_{\text {flu }}$ & $\Phi_{\Delta}$ & $\begin{array}{l}k_{\mathrm{t}}^{\mathrm{PS}} \\
{\left[\mathrm{M}^{-1} \mathrm{~s}^{-1}\right]}\end{array}$ \\
\hline 1 & 538 & 2.5 & 552 & 0.40 & $0.50^{a}$ & $\leq 1.1 \times 10^{8}$ \\
2 & 538 & 7.5 & 554 & 0.33 & $0.57^{a}$ & $\mathrm{ND}$ \\
3 & 542 & 5.1 & 568 & 0.20 & $0.33^{a}$ & $\mathrm{ND}$ \\
4 & 534 & 8.5 & 554 & 0.30 & $0.41^{a}$ & $\mathrm{ND}$ \\
5 & 527 & 4.2 & 576 & 0.33 & $0.61^{a}$ & $5.4 \times 10^{8}$ \\
& & & & & $0.62^{b}$ & \\
6 & 515 & 4.3 & 555 & 0.51 & $0.42^{a}$ & $\leq 2.6 \times 10^{7}$ \\
7 & 533 & 4.3 & 588 & 0.04 & $0.85^{a}$ & $\leq 4.6 \times 10^{7}$ \\
& & & & & $0.82^{b}$ & \\
8 & 426 & 3.9 & 484 & 0.02 & $0.78^{b}$ & $\leq 1.2 \times 10^{7}$ \\
9 & 440 & 3.8 & 521 & 0.05 & $0.96^{b}$ & $\leq 3.9 \times 10^{7}$ \\
10 & 450 & 3.4 & 535 & $<0.01$ & $0.09^{b}$ & $\mathrm{ND}$
\end{tabular}

${ }^{a}$ Reference $\operatorname{RB}$ in $\operatorname{ACN}\left(\Phi_{\Delta}=0.53\right) \cdot{ }^{b}$ Reference PN in ACN $\left(\Phi_{\Delta}=1\right) \cdot{ }^{19}$ ${ }^{c}$ ND not determined.
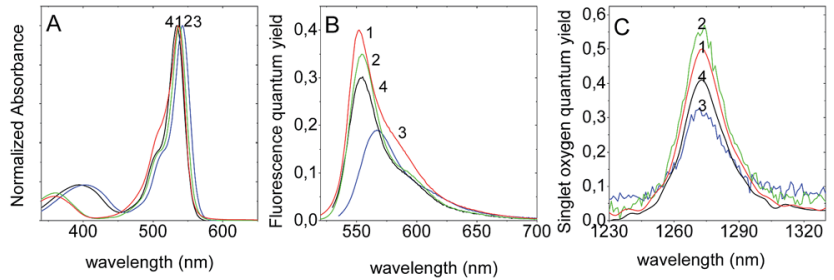

Fig. 1 Aryl-BODIPYs compounds 1-4 (A) normalized absorbance spectra, (B) fluorescence spectra normalized to the fluorescence quantum yield $(C)$ singlet oxygen spectra normalized to the singlet oxygen quantum yield at $\lambda_{\text {exc }}=545 \mathrm{~nm}$.

delocalization and consequently does not practically affect the photophysical properties and the singlet oxygen generation with respect to the unsubstituted 8-position.

Conversely, in the derivatives 3 and $\mathbf{4}$, the aryl groups at meso position can freely rotate $\mathbf{1 1 2}^{\mathbf{2 7}}$ and the internal conversion processes increase to the detriment of the radiative emission from the singlet state and to the intersystem crossing to the triplet state, consequently decreasing both fluorescence and singlet oxygen production.

On the other hand, the position of absorption and emission bands slightly depends on the electronegativity of the different groups attached on the para position of the 8-phenyl (Table 1 and Fig. 1). Generally, with an electron donor substitution at meso position of the BODIPY such as in the case of 8-para-methoxyphenyl compound (4), the LUMO is destabilized and the energy gap between both states increases, producing a blue shift of their respective spectral bands (Fig. 1). However, if the substituent is an electron withdrawing group such as in 8-para-trifluoromethylphenyl (3), the LUMO state is stabilized and the energy gap between both states is lower, giving a red shift of the bands relative to the unsubstituted BODIPY at meso position (Fig. 1). ${ }^{\mathbf{1 5}}$ 
The nature of the group substituted at meso position, i.e. methoxy-phenyl vs. trifluoromethyl-phenyl, has a more significant impact on the fluorescence emission and singlet oxygen generation. Indeed, taking $\mathbf{1}$ as the reference, compound $\mathbf{4}$ with the electron donor $\mathrm{MeO}$ group shows a lower decrease in the efficiency of both processes relative to 3 with the electro withdrawing $\mathrm{CF}_{3}$ group. This effect could be related to the activation of a weakly or non-emissive intra charge-transfer (ICT) process between the BODIPY core and the substituent group at the meso position, process already described in similar compounds. ${ }^{28,29}$ Accordingly, the more activated the ICT state, the less favoured is the radiative fluorescence deactivation from the locally excited (LE) and the intersystem crossing to the triplet state (Fig. S2 in ESI $\uparrow$ ). In fact, according to the Hammett parameters (0.54 and -0.27 , for trifluoromethyl and methoxy groups respectively), ${ }^{30}$ the electron withdrawing character of $\mathrm{CF}_{3}$ is higher than the electron donor ability of the $\mathrm{MeO}$ groups. Consequently, the $\mathrm{CF}_{3}$ substituted derivative would activate in a higher extent the ICT process from the BODIPY core to the aryl substituent than the $\mathrm{MeO}$ substituted compound (in this case the ICT from the aryl group to the BPD core). Nevertheless, the effect of the ICT on the singlet oxygen production will be more clearly seen below in the 8-amino-BODIPY derivatives, in which amines with different electro-donor abilities are substituted at the meso-position of the BODIPY.

From these results it can be concluded that the bromination of the BODIPY core at 2 and 6 positions leads to a balanced contribution of fluorescence emission and singlet oxygen generation. Consequently, both 2,6-dibromo-BODIPYs 1 and 2 show interesting properties according to their dual behaviour and could thus act as theranostic platforms suitable for imaging (fluorescence) and treatment (photodynamic therapy, PDT). The substitution of the aryl group at meso position with no restricted rotation significantly decreases both fluorescence emission and singlet oxygen production due to the increase of internal conversion processes. Depending on the electron donor or electron withdrawing capacity of the groups substituted at meso position, the efficiency of both processes can also be disturbed $\left(\phi_{\mathrm{fl}}\right.$ and $\left.\Phi_{\Delta}\right)$ by the competitive activation of ICT process.

\section{8-Methylthio-BODIPY}

In this subsection, the photophysical properties and the singlet oxygen generation of BODIPYs with a methylthio substituted at meso position but with different halogenated atoms $(5,6$ and 7 in Scheme 1) are studied and compared with the ref. 1.

The halogenation (type and extend) of the BODIPY core strongly influences the position of the absorption and emission bands. In fact, previous studies have confirmed that successive halogenation of the BODIPY core gave rise to a progressive bathochromic shift of both spectral bands..$^{11}$ This is confirmed in the emission spectra of methylthio-BODIPYs with different halide substitutions (Table 1 and Fig. 2B). However, the absorption band for all these compounds is blueshifted relative to $\mathbf{1}$ (Fig. 2A). This blue shift is ascribed to the stabilization of a new resonance form, a hemicyanine-like structure, where the methylthio is delocalized though the

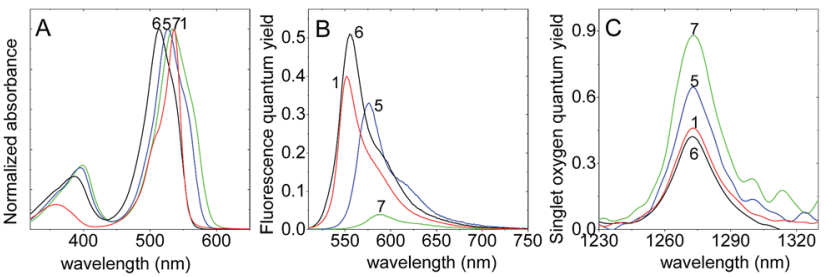

Fig. 2 Methylthio-BODIPY compounds 5-7 (A) normalized absorbance spectra, (B) fluorescence spectra normalized to the fluorescence quantum yield $(C)$ singlet oxygen spectra normalized to the singlet oxygen quantum yield with $\lambda_{\text {exc }}=510 \mathrm{~nm}$.

BODIPY core (resonance form B in Fig. S3, ESI†). ${ }^{17}$ The formation of a hemicyanine-like $\pi$-system has been previously demonstrated for a series of halogen-free BODIPYs with different substitution patterns at meso position. ${ }^{15,29}$ Additionally, the shoulder at lower energies observed in the absorption spectra (Fig. 2) is attributed to the contribution of normal cyanine-like resonance (form A in Fig. S3†) with a more delocalized $\pi$-system. The shorter delocalization of the hemicyanine $\mathrm{B}$ with respect to cyanine-like resonance form A accounts for the blue shift in the absorption spectra, generating a relatively significant Stoke shift. ${ }^{17}$ In this context, since the cyanine-like resonance form is the only species responsible of the emission, the emission bands of all the halogenated methylthio-BODIPYs, even compound 6 with only one bromide atom at 2-position, are red-shifted with respect to the reference compound 1 (Table 1 and Fig. 2). On the other hand, as already stated for non-halogenated BODIPYs,${ }^{15}$ the contribution of the non-emissive hemicyanine-resonance form (methylthio electronically coupled with the BOPIDY core) could be the reason of the lower fluorescence quantum yield in 5 with respect to 1 . As shown in Fig. S3, $\uparrow$ this resonance form is favoured in polar media, such as ACN. For instance, the relative contribution of forms A and B in compound 7, was estimated from the deconvolution of the main absorption band in two Gaussians, in which the component at lower energies (resonance form A) increases around 2.7 times in chloroform with respect to ACN. On the other hand, since the shape of the absorption spectra of 5, 6, and 7 in Fig. 2 is quite similar (i.e. the intensity of the shoulder at lower energies relative to the main absorption band is analogous), the contribution of the resonance forms A and B is probably not very different for all these methylthio-meso BODIPY with different halogenated pattern. On the contrary, the type and extent of halogenation on the BODIPY core will have a more significant impact on the fluorescence capacity and consequently on the singlet oxygen generation due to the heavy atom effect. Thus, compound 7 with two iodine atom substituted at 2,6-position is characterized by a very low fluorescence quantum yield $\left(\phi_{\mathrm{fl}}=0.04\right)$, as a consequence of a strong enhancement of the intersystem crossing relative to 5 with two bromine atoms $\left(\phi_{\mathrm{fl}}=0.33\right)$ or to the mono-brominated $6\left(\phi_{\mathrm{fl}}=0.51\right)$ (Fig. 2 and Table 1$)$. As expected, the di-iodo compound 7 shows the highest singlet oxygen quantum yield, $\Phi_{\Delta}=0.82-0.85$ (Table 1) since the spin-orbit coupling depends on the fourth power of the atoms 
orbital quantum number. The substitution by two iodine atoms thus induces a more efficient ISC relative to the substitution by two bromine atoms $\left(5, \Phi_{\Delta}=0.61-0.62\right)$, or even with respect to the compound with only one bromine atom (6, $\left.\Phi_{\Delta}=0.42\right)$.

The more relevant aspect in these methylthio-BODIPY compounds, is that owing to their relatively intense $\mathrm{S}_{0}-\mathrm{S}_{2}$ absorption band (molar extinction coefficient $\varepsilon \sim 1.3 \times 10^{4} \mathrm{M}$ $\mathrm{cm}^{-1}$, Fig. 2A), the determination of the singlet oxygen quantum yield is possible by the direct method exciting either in the UV (with phenalenone as reference) or in the green-yellow visible range (using Rose Bengal as standard, Table 2). Actually, similar singlet oxygen quantum yields were obtained in ACN for these methylthio-BODIPYs with either reference. This result in turn also validates the singlet oxygen quantum yield determined for RB in $\operatorname{ACN}\left(\Phi_{\Delta}=0.53\right)$, for which very scattered values $\left(\Phi_{\Delta}=0.42-0.83\right)$ may be found in the literature. ${ }^{33-35}$

In the following, the analysis of singlet oxygen quantum yields of 7 in several solvents of various polarities was addressed. It should be stressed here that the solvent polarity is not the only factor governing singlet oxygen quenching and lifetimes and that other parameters dealing with the formation and stability of the collision complex between a solvent molecule $\mathrm{S}$ and oxygen $\left(\mathrm{S}-\mathrm{O}_{2}\right)$, out of the scope of the present paper, have to be considered. ${ }^{32}$ Nevertheless, the singlet oxygen quantum yield of compound 7 in ACN, THF, dichloromethane $(\mathrm{DCM})$ or chloroform are very similar $\left(\Phi_{\Delta(\mathrm{PN})}=0.79-0.82\right.$, Table 2) when phenalenone (PN) is used as reference. However, when $\mathrm{RB}$ in $\mathrm{ACN}$ is used as standard, greater discrepancies are observed, with an over estimation of the singlet oxygen quantum yields $\left(\Phi_{\Delta(\mathrm{RB})}=1.15-1.31\right)$, particularly in chlorinated solvents, while consistent values with the previous ones are obtained in ACN or THF $\left(\Phi_{\Delta(\mathrm{RB})}=0.82-0.85\right)$. This result highlights the already mentioned more difficult correction to be made when using different solvents between the reference and the PS under investigation (eqn SI 07, ESI $\dagger$ ). In other words, since RB cannot be used as a reference in $\mathrm{DCM}$ or $\mathrm{CHCl}_{3}$ due to its poor solubility, it is highly possible that the singlet oxygen quantum yield measured for 7 using RB in ACN as the standard are poorly reliable. The sole consistent result to consider here is in the same solvent, i.e. $\mathrm{ACN}\left(\Phi_{\Delta(\mathrm{RB})}=0.85\right)$.

Moreover the rate constants of the total quenching of singlet oxygen by the PS itself in ACN, $k_{\mathrm{t}}^{\mathrm{PS}}\left(\mathrm{M}^{-1} \mathrm{~s}^{-1}\right.$ ) (see eqn SI 04 in

Table 2 Singlet oxygen quantum yield of compound 7 and lifetime of ${ }^{1} \mathrm{O}_{2}$ in different solvents

\begin{tabular}{lllll}
\hline Solvent & $E_{\mathrm{T}}(30)^{a}$ & $\Phi_{\Delta(\mathrm{PN})}{ }^{b}$ & $\Phi_{\Delta(\mathrm{RB})}{ }^{c}$ & $\tau_{\Delta}{ }^{d}(\mu \mathrm{s})$ \\
\hline THF & 37.4 & 0.81 & 0.82 & $23(20)$ \\
$\mathrm{CHCl}_{3}$ & 39.1 & 0.80 & 1.31 & $201(229)$ \\
DCM & 40.7 & 0.79 & 1.15 & $97(99)$ \\
ACN & 45.6 & 0.82 & 0.85 & $81(77)$
\end{tabular}

${ }^{a} E_{\mathrm{T}}(30)$ is the Reichardt parameter that indicates the polarity of the solvents. ${ }^{b}$ PN as reference in each solvent assuming $\Phi_{\mathrm{PN}}=1$ with $\lambda_{\text {exc }}$ $=375 \mathrm{~nm} .{ }^{c} \mathrm{RB}$ as reference in $\operatorname{ACN}\left(\Phi_{\mathrm{RB}}=0.53\right)$ with $\lambda_{\text {exc }}=510 \mathrm{~nm}$. ${ }^{d}$ Values between brackets from literature. ${ }^{31,32}$
$\mathrm{ESI} \dagger$ for the details on calculation), were determined for these methylthio-BODIPYs by varying the PS concentration relative to the reference (Rose Bengal). The independence of the $\left(\mathrm{S}_{\mathrm{e}}^{\mathrm{R}} /\right.$ $\left.\mathrm{S}_{\mathrm{e}}^{\mathrm{Ps}}\right)\left(\alpha^{\mathrm{Ps}} / \alpha^{\mathrm{R}}\right)$ with respect to the concentration for 1,6 and 7 (Fig. 3A stars, triangles and squares, respectively) indicates that the quenching of ${ }^{1} \mathrm{O}_{2}$ by these BODIPYs was negligible compared to the quenching by the solvent $\left(k_{\mathrm{t}}^{\mathrm{PS}}[\mathrm{PS}] \ll k_{\mathrm{d}}\right)$. On the other hand, a much more significant $k_{\mathrm{t}}^{\mathrm{PS}}=5.4 \times 10^{8} \mathrm{M}^{-1} \mathrm{~s}^{-1}$ is obtained for compound 5 (Fig. 3A rhombus).

In order to confirm these results, the ${ }^{1} \mathrm{O}_{2}$ specific chemical probe, 9,10-dimethylanthracene (DMA), is added to the photosensitizer solution in ACN. In this case, the singlet oxygen generation can be followed photometrically since the characteristic absorption bands in the 300-400 $\mathrm{nm}$ range of DMA will gradually disappear due to the formation of the endoperoxide as the reaction product ${ }^{36}$ (Fig. 3B). First the intensity of the absorption band of 7 is constant during the reaction time, confirming the stability against photobleaching on the timescale of the experiment. A relatively fast decrease of the absorption bands of DMA with time is observed and the singlet oxygen quantum yield calculated by this approach (eqn SI 08-11 in ESI $\dagger$ ), using $k_{\mathrm{t}}^{\mathrm{Q}}=8.8 \times 10^{7} \mathrm{M}^{-1} \mathrm{~s}^{-1}$ and $k_{\mathrm{r}}=5.3 \times$ $10^{7} \mathrm{M}^{-1} \mathrm{~s}^{-1}$ values for DMA in ACN found in the literature ${ }^{37,38}$ is $\Phi_{\Delta} \sim 0.75$. This value is in good agreement with the quantum yield determined by the direct method given the possible errors on the kinetic rate constants from the literature.

From all these results, it appears that compound 7 could be further analysed as a suitable solvent-independent standard for the UV and green-yellow region of the vis spectra, instead of the usual RB standard, with a possible use in chlorinated solvents such as $\mathrm{CH}_{2} \mathrm{Cl}_{2}$ or $\mathrm{CHCl}_{3}$ where $\mathrm{RB}$ is not soluble. A more detailed study of compound 7 as potential reference should be addressed in an additional work, out of the scope of the present paper, dealing with the complete photophysical characterization of the excited states of 7 both from an experimental and theoretical point of view. As a matter of fact, for the other well-known standard photosensitizer phenalenone, an extensive discussion (summarized in ref. 4) of the intersystem crossing efficiency between the $\mathrm{S}$ and $\mathrm{T}$ states allows understanding the insensitivity of singlet oxygen quantum yields to solvent polarity.
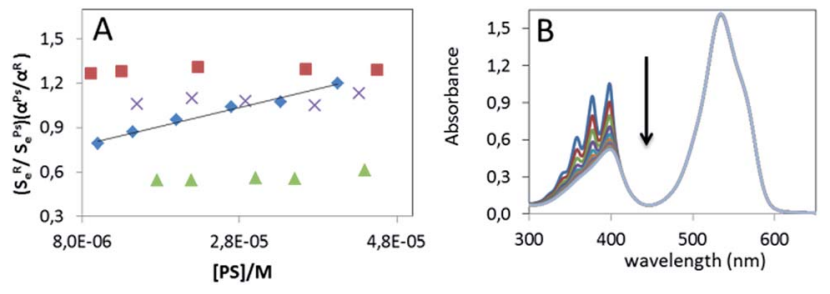

Fig. 3 (A) Variation of the ratio of the ${ }^{1} \mathrm{O}_{2}$ emission signal for the reference and BOPIDY as a function of the BODIPY concentration in ACN at room temperature: 1 (stars), 6 (squares), 7 (triangles) and 5 (rhombus). Errors: approx 10\%. (B) Evolution of the absorption spectra of a solution containing BODIPY 7 and DMA during 15 min upon 547 $\mathrm{nm}$ excitation. 


\section{8-Amino-BODIPY}

In this section, the photophysical properties and the singlet oxygen generation of BODIPYs with two iodine atoms at 2,6 position and various substituted amino groups at meso position (8, 9 and 10, Scheme 1) were studied.

As commented above, the position of the spectral bands markedly depends on the heteroatom attached at the meso position of the BODIPY and more particularly on the electronegativity of that heteroatom. That is, the larger electron donor capacity of the group at meso position, the higher the hypsochromic shift because the hemicyanine resonance structure (Fig. S5 $\dagger$ ) is more favoured. ${ }^{15,17,39}$

In fact, BODIPY 8 with a NHMe substitution at meso position gives a very pronounced blue shift, more than $100 \mathrm{~nm}$ (Table 1 and Fig. 4), with respect to 7 (methylthio at meso position and same halogenation pattern), in agreement with their respective Hammett parameters $\left(\sigma_{\mathrm{p}}^{+}=-\mathbf{1 . 8 1}\right.$ for NHMe and $\sigma_{\mathrm{p}}^{+}=-\mathbf{0 . 6 0}$ for $\mathrm{SMe}$, respectively). ${ }^{\mathbf{3 0}}$

The fluorescence spectra of these amino substituted iodoBPDs show a very poor emission band $\left(\phi_{\mathrm{fl}} \leq 0.05\right)$ with respect to the analogous compounds without any heavy atom $\left(\phi_{\mathrm{fl}} \sim 0.80\right.$ in ethanol $\left.{ }^{5}\right)$. This is attributed to the presence of halogen atoms, which enhance the intersystem crossing (ISC) process to the triplet state. However, in these halogenated BODIPYs, the competitive formation of an ICT state, which strongly depends on the amine-group substitution pattern at the meso position, will have a higher impact on the singlet oxygen production than on their fluorescence. Indeed, the replacement of the methyl group $\left(\sigma_{\mathrm{p}}^{+}=-0.31\right)$ in 8 by a propargyl group in 9 (lower electron-donor strength, $\sigma_{\mathrm{p}}^{+}=+0.18$ ), disfavours the activation of ICT and consequently increases the singlet oxygen production $\left(\Phi_{\Delta}=0.96\right.$, Table 1$)$ with respect to compound $8\left(\Phi_{\Delta}=0.78\right)$.

As a result, both $\mathbf{8}$ and $\mathbf{9}$ represent an interesting alternative as photosensitisers for singlet oxygen production with high quantum yields upon blue visible light and UV excitation. Moreover, the ${ }^{1} \mathrm{O}_{2}$ production by those amino-BODIPY is independent of their concentration (Fig. S6†), indicating a negligible self-quenching of singlet oxygen $\left(k_{\mathrm{t}}^{\mathrm{PS}}[\mathrm{PS}] \ll k_{\mathrm{d}}\right)$.

On the other hand, although tertiary amines have higher electron-donating character, the hypsochromic shift of $\mathbf{1 0}$ relative to 7 is smaller than that observed for the secondary amines $\mathbf{8}$ and 9. In this particular case, the cyclic amine is not in a coplanar
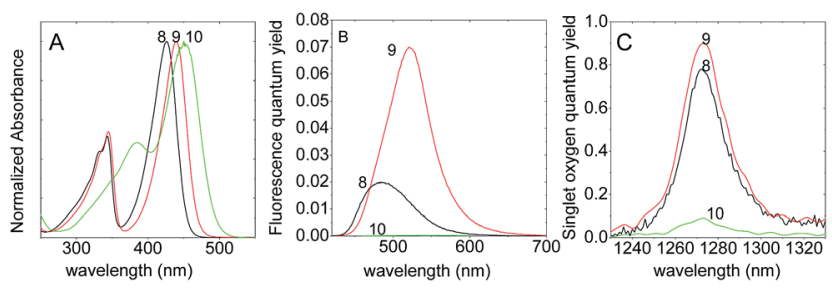

Fig. 4 The amino-BODIPY compounds 8-10 (A) normalized absorbance spectra (B) fluorescence spectra normalized to the fluorescence quantum yield (C) singlet oxygen spectra normalized to the singlet oxygen quantum yield with $\lambda_{\text {exc }}=400 \mathrm{~nm}$. arrangement with the BODIPY core due to steric hindrance, reducing the probability of the formation of the hemicyanine (Fig. S5 $\dagger$ ), and consequently the shift of the spectral bands to the blue (Fig. 4). Moreover, the reason why 10 does not show any significant singlet oxygen generation is likely due to the fee rotation of the amine group which enhances the internal conversion process, to the detriment of intersystem crossing to the triplet state and consequently to singlet oxygen generation, as previously commented for the aryl-BODIPY $\mathbf{3}$ and $\mathbf{4}$ for instance. In fact, as a consequence of these motions, a twisted intramolecular charge transfer state (TICT) may also be favoured, which will compete with the radiative emission from the singlet state (fluorescence) and intersystem process to the triplet state (singlet oxygen production). Therefore, the substitution of piperidine group at the meso position in not recommended.

\section{Conclusions}

First, the type and extent of halogenation on the BODIPY core have a significant impact on the fluorescence and singlet oxygen quantum yields due to the heavy atom effect. Thus, by bromination of the BODIPY core (at 2-position or both 2,6positions), the chromophores exhibit a dual functionality since they maintain both a high enough fluorescence emission $\left(\phi_{\mathrm{fl}} \sim\right.$ 0.40-0.30) suitable for bioimaging but, at the same time, a high enough triplet state population to promote singlet oxygen generation $\left(\Phi_{\Delta} \sim 0.50-0.60\right)$ for PDT. A balanced efficiency is thus achieved, which could be important for theranosis application. On the other hand, by iodination of the BOPIDY at 2,6 positions, the spin orbit coupling is enhanced and very high singlet oxygen quantum yield are obtained $\left(\Phi_{\Delta} \sim 0.80-\right.$ $0.90)$ at the expense of the fluorescence efficiency $\left(\phi_{\mathrm{fl}}<0.10\right)$. In vitro experiments will be carried out with the present compounds to test their internalization into the cell, their efficiency to kill tumor cells together with their toxicity in dark conditions.

On the other hand, the substitution patterns at the meso position of the BODIPY plays an important role in the deactivation pathways of the first singlet excited state, such as internal conversion and possible internal charge transfer processes. Thus, the substitution of groups with free rotational motions (such as phenyl and piperidine groups) deactivates both fluorescence and singlet oxygen generation due to the increase of internal conversion processes. Moreover, the electron-donor strength of the substituent at the meso position (especially when including heteroatoms) not only modifies the position of the spectral bands but also strongly affects the fluorescence and singlet oxygen quantum yields. In this regard, substituents with relatively high electron-donor strength such as NHMe, will activate an ICT state reducing the efficiency of the singlet oxygen generation.

At last, a new standard for singlet oxygen quantum yield determination based on an iodo-BODIPY with a methylthio at its meso position (compound 7) is proposed. This compound shows intense bands in the visible $\left(\lambda_{\mathrm{abs}}=533 \mathrm{~nm}, \varepsilon_{\max }>40000\right.$ $\left.\mathrm{M}^{-1} \mathrm{~cm}^{-1}\right)$ and in the UV range $\left(\lambda_{\text {abs }}=400 \mathrm{~nm}, \varepsilon_{\max }>10000\right.$ $\mathbf{M}^{-1} \mathrm{~cm}^{-1}$ ), together with high singlet oxygen quantum yields 
$\left(\Phi_{\Delta} \sim 0.8\right)$, practically solvent-independent in a broad polarity range. Moreover, no noticeable quenching of ${ }^{1} \mathrm{O}_{2}$ by this PS was observed $\left(k_{\mathrm{t}}^{\mathrm{PS}}[\mathrm{PS}] \ll k_{\mathrm{d}}\right)$. Other favorable chemico-physical features already demonstrated for BODIPY-dyes (i.e. low photobleaching and high lipophilicity) make this particular BODIPY a possible alternative as a standard photosensitizer for UV and visible range, in a large variety of solvents, including chlorinated ones, even if further work is needed to firmly establish it as a universal reference.

\section{Acknowledgements}

The authors thank the Spanish Ministry of Economy and Competitiveness, MINECO, (MAT 2014-51937-C3-3) and Basque Government (IT339-10 and IT912-16) for funding this research. VMM acknowledges Ministerio de Economía y Competitividad MINECO for 'Ramón y Cajal' contract (RYC-2011-09505). NEE acknowledges Universidad del País Vasco (UPV-EHU) and Université de Pau et Pays de l'Adour (UPPA) for a co-advised doctoral grant.

\section{Notes and references}

1 J. Chem, L. Keltner, J. Christophersen, F. Zheng, M. Krouse, A. Singhal and S.-S. Wang, Cancer J., 2002, 8, 154-163.

2 S. G. Awuah and Y. You, RSC Adv., 2012, 2, 11169.

3 M. C. DeRosa and R. J. Crutchley, Coord. Chem. Rev., 2002, 233-234, 351-371.

4 D. García-Fresnadillo and S. Lacombe, Reference Photosensitizers for the Production of Singlet Oxygen in Singlet Oxygen: Applications in Biosciences and Nanosciences, ed. S. Nonell and C. Flors, The Royal Society of Chemistry, 2016, vol. 1 .

5 Y. C. Lai, S. Y. Su and C. C. Chang, ACS Appl. Mater. Interfaces, 2013, 5, 12935-12943.

6 A. Kamkaew, S. H. Lim, H. B. Lee, L. V. Kiew, L. Y. Chung and K. Burgess, Chem. Soc. Rev., 2012, 42, 77-88.

7 A. E. O'Connor, W. M. Gallagher and A. T. Byrne, Photochem. Photobiol., 2009, 85, 1053-1074.

8 T. Yogo, Y. Urano, Y. Ishitsuka, F. Maniwa and T. Nagano, J. Am. Chem. Soc., 2005, 127, 12162-12163.

9 J. Karolin, L. B. a. Johansson, L. Strandberg and T. Ny, J. Am. Chem. Soc., 1994, 116, 7801-7806.

10 J. Zhao, W. Wu, J. Sun and S. Guo, Chem. Soc. Rev., 2013, 42, 5323-5351.

11 M. J. Ortiz, A. R. Agarrabeitia, G. Duran-Sampedro, J. Bañuelos Prieto, T. Arbeloa López, W. A. Massad, H. A. Montejano, N. A. García and I. López Arbeloa, Tetrahedron, 2012, 68, 1153-1162.

12 R. Sharma, H. B. Gobeze, T. Chatterjee, P. A. Karr, F. D'Souza and M. Ravikanth, RSC Adv., 2016, 6, 24111-24114.

13 S. G. Awuah and Y. You, RSC Adv., 2012, 2, 11169-11183.

14 S. H. Lim, C. Thivierge, P. Nowak-Sliwinska, J. Han, H. Van Den Bergh, G. Wagnières, K. Burgess and H. B. Lee, J. Med. Chem., 2010, 53, 2865-2874.

15 I. Esnal, I. Valois-Escamilla, C. F. a. Gómez-Durán, A. UríasBenavides, M. L. Betancourt-Mendiola, I. López-Arbeloa,
J. Bañuelos, I. García-Moreno, A. Costela and E. PeñaCabrera, ChemPhysChem, 2013, 14, 4134-4142.

16 C. F. A. Gómez-Durán, I. Esnal, I. Valois-Escamilla, A. UríasBenavides, J. Bañuelos, I. López-Arbeloa, I. García-Moreno and E. Peña-Cabrera, Chem.-Eur. J., 2015, 21, 1-15.

17 I. Esnal, A. Urías-Benavides, C. F. A. Gómez-Durán, C. a. Osorio-Martínez, I. García-Moreno, A. Costela, J. Bañuelos, N. Epelde, I. López Arbeloa, R. Hu, B. Z. Tang and E. Peña-Cabrera, Chem.-Asian J., 2013, 8, 2691-2700.

18 F. Wilkinson, W. P. Helman and A. B. Ross, J. Phys. Chem. Ref. Data, 1993, 22, 113-262.

19 C. G. Martinez, A. Neumer, C. Marti, S. Nonell, A. M. Braun and E. Oliveros, Helv. Chim. Acta, 2003, 86, 384-397.

20 H. Kotani, K. Ohkubo and S. Fukuzumi, J. Am. Chem. Soc., 2004, 126, 15999-16006.

21 S. Mathai, T. a. Smith and K. P. Ghiggino, Photochem. Photobiol. Sci., 2007, 6, 995-1002.

22 F. Li, S. I. Yang, Y. Ciringh, J. Seth, C. H. Martin, D. L. Singh, D. Kim, R. R. Birge, D. F. Bocian, D. Holten and J. S. Lindsey, J. Am. Chem. Soc., 1998, 120, 10001-10017.

23 F. López Arbeloa, J. Bañuelos, V. Martínez, T. Arbeloa and I. López Arbeloa, Int. Rev. Phys. Chem., 2005, 24, 339-374.

$24 \mathrm{H}$. L. Kee, C. Kirmaier, L. Yu, P. Thamyongkit, W. J. Youngblood, M. E. Calder, L. Ramos, B. C. Noll, D. F. Bocian, W. R. Scheidt, R. R. Birge, J. S. Lindsey and D. Holten, J. Phys. Chem. B, 2005, 109, 20433-20443.

25 M. A. H. Alamiry, A. C. Benniston, G. Copley, K. J. Elliott, A. Harriman, B. Stewart and Y. G. Zhi, Chem. Mater., 2008, 20, 4024-4032.

26 L. G. S. Brooker, A. C. Craig, D. W. Heseltine, P. W. Jenkins and L. L. Lincoln, J. Am. Chem. Soc., 1965, 87, 2443-2450.

27 J. Bañuelos, I. J. Arroyo-Córdoba, I. Valois-Escamilla, A. Alvarez-Hernández, E. Peña-Cabrera, R. Hu, B. Z. Tang, I. Esnal, V. Martínez and I. López Arbeloa, RSC Adv., 2011, 1, 677-684.

28 M. Kollmannsberger, K. Rurack, U. Resch-Genger and J. Daub, J. Phys. Chem. A, 1998, 102, 10211-10220.

29 J. Bañuelos, V. Martín, C. F. A. Gómez-Durán, I. J. A. Córdoba, E. Peña-Cabrera, I. García-Moreno, Á. Costela, M. E. Pérez-Ojeda, T. Arbeloa and Í. L. Arbeloa, Chem.-Eur. J., 2011, 17, 7261-7270.

30 C. Hansch, a. Leo and R. W. Taft, Chem. Rev., 1991, 91, 165195.

31 A. Fallis, J. Am. Chem. Soc., 1982, 104, 2065-2067.

32 C. Schweitzer and R. Schmidt, Chem. Rev., 2003, 103, 16851757.

33 F. Ronzani, A. Trivella, E. Arzoumanian, S. Blanc, M. Sarakha, C. Richard, E. Oliveros and S. Lacombe, Photochem. Photobiol. Sci., 2013, 12, 2160-2169.

34 R. Schmidt and E. Afshari, J. Phys. Chem., 1990, 94, 43774378.

35 Y. Usui, H. Koike and Y. Kurimura, Bull. Chem. Soc. Jpn., 1987, 60, 3373-3378.

36 B. F. Sels, D. E. De Vos, P. J. Grobet, F. Pierard, F. K.-D. Mesmaeker and A. J. Pierre, J. Phys. Chem. B, 1999, 103, 11114-11123. 
37 G. S. Günther, E. M. Lemp and A. L. Zanocco, in Boletín de la Sociedad Chilena de Química, 2000, pp. 637-644.

38 I. Gutiérrez, S. G. Bertolotti, M. A. Biasutti, A. T. Soltermann and N. A. García, Can. J. Chem., 1997, 75, 423-428.
39 C. Osorio-Martínez, A. Urías-Benavides, A. Gómez-Durán, J. Bañuelos, I. Esnal, I. López-Arbeloa and E. Peña-Cabrera, J. Org. Chem., 2012, 77, 5434-5438. 